\title{
A parity function for studying the molecular electronic structure
}

\section{Schmider, Hartmut}

Published in:

Journal of Chemical Physics

Link to article, DOI:

$10.1063 / 1.472913$

Publication date:

1996

Document Version

Publisher's PDF, also known as Version of record

Link back to DTU Orbit

Citation (APA):

Schmider, H. (1996). A parity function for studying the molecular electronic structure. Journal of Chemical Physics, 105(24), 11134-11142. https://doi.org/10.1063/1.472913

\section{General rights}

Copyright and moral rights for the publications made accessible in the public portal are retained by the authors and/or other copyright owners and it is a condition of accessing publications that users recognise and abide by the legal requirements associated with these rights.

- Users may download and print one copy of any publication from the public portal for the purpose of private study or research.

- You may not further distribute the material or use it for any profit-making activity or commercial gain

- You may freely distribute the URL identifying the publication in the public portal

If you believe that this document breaches copyright please contact us providing details, and we will remove access to the work immediately and investigate your claim. 


\title{
A parity function for studying the molecular electronic structure
}

\author{
Hartmut Schmider ${ }^{\mathrm{a})}$ \\ Department of Chemistry, Chemical Physics, Technical University of Denmark, DTU-207, 2800 Lyngby, \\ Denmark
}

(Received 6 August 1996; accepted 9 September 1996)

\begin{abstract}
Sections through the molecular Wigner function with zero momentum variable are shown to provide important information about the off-diagonal regions of the spinless one-particle reduced density matrix. Since these regions are characteristic for the bonding situation in molecules, the sections are qualitatively even more affected by the presence of chemical bonds than a complementary projection, the reciprocal form factor. In this paper we discuss, on the grounds of a variety of examples, how this rather simple function may aid the understanding of the chemical bond on a one-particle level. () 1996 American Institute of Physics. [S0021-9606(96)02846-2]
\end{abstract}

\section{INTRODUCTION}

The central quantity for the analysis of a chemical system on a one-particle level is the reduced one-particle density operator. Originally introduced by Dirac, ${ }^{1}$ this quantity was further refined and generalized by Husimi ${ }^{2}$ and made a fundamental concept in theoretical chemistry by Löwdin ${ }^{3}$ and McWeeny. ${ }^{4,5}$ In its representation in terms of atomcentered one-particle basis functions, it has entered the pool of standard quantities to be computed in electronic-structure calculations, under the name of "charge and bond-order matrix." ${ }^{6}$ Less attention has been devoted to representations in either position or momentum space. It was pointed out by Weyrich $^{7,8}$ that the direct analysis of the position representation $\rho\left(\vec{r}, \vec{r}^{\prime}\right)$ is beneficial, particularly for molecular systems, because of characteristic non-diagonal regions and their close relationship to the momentum density $\pi(\vec{p}){ }^{7,9,10}$ The latter is, in principle, reconstructible from experimental properties, such as the Compton profile $J(\vec{q}) .{ }^{11}$ A small number of studies on the structure of the (spin traced) single-particle density matrix (SPDM) has appeared in recent years ${ }^{8,12-16}$ and attempted to relate features of covalent bonds to patterns in its off-diagonal regions. Direct studies are naturally hampered by the difficulties in visualizing a function of six independent variables, and one has to revert to sections or projections. In this paper, a projection of the SPDM is introduced that is deemed helpful for interpreting molecular systems in terms other than the conventional ones of charge or momentum density.

\section{THE SPDM IN POSITION SPACE, AND TWO PROJECTIONS OF IT}

As a projector onto the space of one-particle functions, the one-particle density operator takes, of course, different forms depending on the representation into which it is cast. Although momentum-space studies have been conducted in increasing number since the pioneer work of Coulson and co-workers, ${ }^{17}$ position (or "direct") space is certainly the more intuitive representation, in terms of which "the nature

\footnotetext{
a)Present address: Department of Chemistry, Queen's University, Kingston, Ontario K7L 3N6, Canada.
}

of the chemical bond" may be discussed. In the following, we will restrict ourselves to position space, although occasional references to the complementary momentum space will suggest themselves.

The common definition of the SPDM is as a sequence of reductions from the full many-particle density matrix $\Gamma$

$$
\begin{aligned}
\rho\left(\vec{r}, \vec{r}^{\prime}\right)= & N \int \ldots \int \Gamma\left(\vec{x}, \vec{x}_{2} \ldots \vec{x}_{N} ; \vec{x}^{\prime}, \vec{x}_{2} \ldots \vec{x}_{N}\right) \\
& \times \delta\left(\sigma, \sigma^{\prime}\right) \mathrm{d} \vec{x}_{2} \ldots \mathrm{d} \vec{x}_{N} \mathrm{~d} \sigma \mathrm{d} \sigma^{\prime},
\end{aligned}
$$

where the combined space and spin variables are denoted by $\vec{x}=(\vec{r}, \sigma)$, and spin summations are written as integrals.

This definition implies that all coordinates but one are of no interest, and that electrons are indistinguishable. Equating the two independent coordinates $\vec{r}$ and $\vec{r}^{\prime}$, will yield the familiar charge density $\rho(\vec{r})$, which is, employing the normalization used above, a number density, i.e., $\rho(\vec{r}) \mathrm{d} \vec{r}$ gives the expected number of electrons in a volume element $\mathrm{d} \vec{r}$ centered at $\vec{r}$. At this point it is helpful to change the coordinate system from symmetric coordinates (in the following, we will use $\vec{r}^{\prime}$ and $\vec{r}^{\prime \prime}$ to denote those) to ones that label the geometric center $\vec{r}$ and the separating vector $\vec{s}$ (see, e.g., Refs. 16, 18);

$$
\vec{r}=\left(\vec{r}^{\prime}+\vec{r}^{\prime \prime}\right) / 2 \text { and } \vec{s}=\left(\vec{r}^{\prime \prime}-\vec{r}^{\prime}\right) .
$$

Such a change of variables is employed as a standard in the analysis of pair densities, and leads there to the definition of intracule and extracule densities (see, e.g., Refs. 19, 20). It is important to not confuse the similar treatment here, and we will call $\vec{r}$ the external, and $\vec{s}$ the internal coordinate in the following. The charge density $\rho(\vec{r})$ may now be written as an initial value of the SPDM in these new coordinates

$$
\widetilde{\rho}(\vec{r}, \vec{s})=\rho\left(\vec{r}^{\prime}, \vec{r}^{\prime \prime}\right), \quad \rho(\vec{r})=\widetilde{\rho}(\vec{r}, 0) .
$$

The charge density does not account for nonlocal or nondiagonal regions in the SPDM, since the separation vector $\vec{s}$ is zero. However, the main impact of the presence of covalent bonding has been shown to be concentrated exactly in those regions. ${ }^{7,8,14}$ A quantity that depends critically on the non-diagonal parts of the SPDM is the reciprocal form factor 
$B(\vec{s})$, which may alternatively be defined as a weighted sum over " "autocorrelation functions" of the natural orbitals ${ }^{7,9}$ (the latter are the eigenfunctions of the SPDM, see Ref. 3 for a detailed treatment), or the Fourier-transform of the momentum density $\pi(\vec{p})$ (the latter being the analogue of the charge density in the momentum representation)

$$
B(\vec{s})=\int \widetilde{\rho}(\vec{r}, \vec{s}) d \vec{r}=\int \pi(\vec{p}) \mathrm{e}^{-i \vec{p} \cdot \vec{s}} d \vec{p}
$$

The reciprocal form factor has become a standard tool for the interpretation of Compton profiles and momentum densities in position space, and has found widespread application in the reconstruction of the latter (e.g., Ref. 21). However, since the external variable $\vec{r}$ has been integrated out in the definition (4), no information about the locality is left, and $B(\vec{s})$ is completely equivalent to the momentum density.

If a simultaneous representation of the SPDM in terms of position and momentum variables is desired, one has to recourse to the classical notion of a phase-space distribution. This path is riddled with difficulties arising from the quantum-mechanical nature of the systems studied, and phase-space functions can neither be unique, nor interpretable in probabilistic terms. There exists a representation that is a simple Fourier transform of $\widetilde{\rho}(\vec{r}, \vec{s})$, and that is preferable to other ways of introducing classical concepts into quantum mechanics. ${ }^{2-24}$ The resulting function is called the Wigner function, and was introduced by its namesake in $1932 .{ }^{23}$ The definition is

$$
W(\vec{r}, \vec{p})=(2 \pi)^{-3} \int \tilde{\rho}(\vec{r}, \vec{s}) \mathrm{e}^{i \vec{s} \cdot \vec{p}} d \vec{s}
$$

Atomic units are used throughout, i.e., $\hbar=e=m_{e}=1$, and consequently proportionality factors of $\hbar$ are omitted. Note that the variable $\vec{p}$ is the external coordinate in momentum space, i.e., it serves the same purpose as $\vec{r}$ in position space. This implies that local quantities in one space are connected by Fourier transformations with nonlocal ones in the other. One may speculate about the physical meaning of the Wigner function (see, e.g., Refs. 25, 26), particularly since it shows large negative areas for most systems, and therefore cannot be called a density. This is easily understood if one recalls that the Heisenberg principle states that a particle cannot simultaneously be assigned a definite position and momentum, and that the notion of a probability of finding a particle at an exact point in phase-space is therefore meaningless on a quantum-mechanical level. On the other hand, operationally the Wigner function may serve as a weight function for phase-space functions that correspond (via the Wigner-Weyl rules) to a given operator, ${ }^{24}$ and maybe that is the only real meaning it has. However, integration over either $\vec{r}$ or $\vec{p}$ yields the densities in the space of the remaining variable as marginals

$$
\int W(\vec{r}, \vec{p}) d \vec{p}=\rho(\vec{r}) ; \quad \int W(\vec{r}, \vec{p}) d \vec{r}=\pi(\vec{p})
$$

Another interpretation of $W(\vec{r}, \vec{p})$ was given originally by Grossmann ${ }^{27}$ and Royer: ${ }^{25}$ The Wigner function may be seen as the expectation value of a parity operator in phase-space (see also Ref. 28). In terms of the natural orbitals, this means that if an orbital is completely inversion symmetric about a point $(\vec{r}, \vec{p})$, it will contribute $\pi^{-3}$ to the Wigner function at that point, and if it is antisymmetric, the contribution will be $-\pi^{-3}$. This fact was first pointed out by Dahl and Springborg $^{29,30}$ (see also Ref. 31).

To return to the question of interpreting the SPDM in position space; if the momentum variable $\vec{p}$ is set to zero in $W(\vec{r}, \vec{p})$, i.e., if an initial value is taken in Eq. (5), the result is proportional to an integration of the SPDM $\widetilde{\rho}(\vec{r}, \vec{s})$ over the internal variable $\vec{s}$, rather than $\vec{r}$ for the reciprocal form factor. This is, of course, also a projection of the nondiagonal regions of the SPDM, but over a subspace orthogonal to the one in the definition (4) of $B(\vec{s})$

$$
P(\vec{r})=W(\vec{r} ; 0)=(2 \pi)^{-3} \int \widetilde{\rho}(\vec{r}, \vec{s}) d \vec{s}
$$

The resulting function $P(\vec{r})$ will, in the following, be called " "parity function,', and it includes a good deal of information about the nonlocal regions of the SPDM, in a local fashion. Note that the normalization in Eq. (7) differs from the one in Eq. (4) by a factor of $(2 \pi)^{-3}$. We retain this factor in order to keep the compatibility with the Wigner function. Owing to the definition of the latter, this parity function integrates to the value of the momentum density at the origin of momentum space. As we will show, it can be quite helpful for visualizing the bond situation in molecules.

The somewhat confusing situation will be explained in more detail in the following section using examples of onedimensional "diatomic" systems. A good introduction is also given in Ref. 18. The principles are illustrated by Fig. 1. Whereas $B(\vec{s})$ results from an integration parallel to the diagonal of the density matrix, i.e., over the external coordinate $\vec{r}, P(\vec{r})$ is obtained by integrating perpendicular to this diagonal, i.e., over the internal coordinate $\vec{s}$.

To summarize; in order to reduce the number of variables in the SPDM, and nevertheless retain some information about nonlocal contributions (which are important for understanding bonding in molecules), we focus on two projections of the SPDM. The well-known external projection in $(\vec{r}, \vec{s})$ variables yields the reciprocal form factor, retaining information equivalent to the momentum density, and therefore losing all notion of absolute (as opposed to relative) position. The other internal one, is linked to a zero-momentum section through the Wigner distribution, and therefore sacrifices almost all information about momentum space ${ }^{32}$ for the benefit of a local description of the parity of the SPDM. In the following sections, we will examine the latter using some simple examples. 

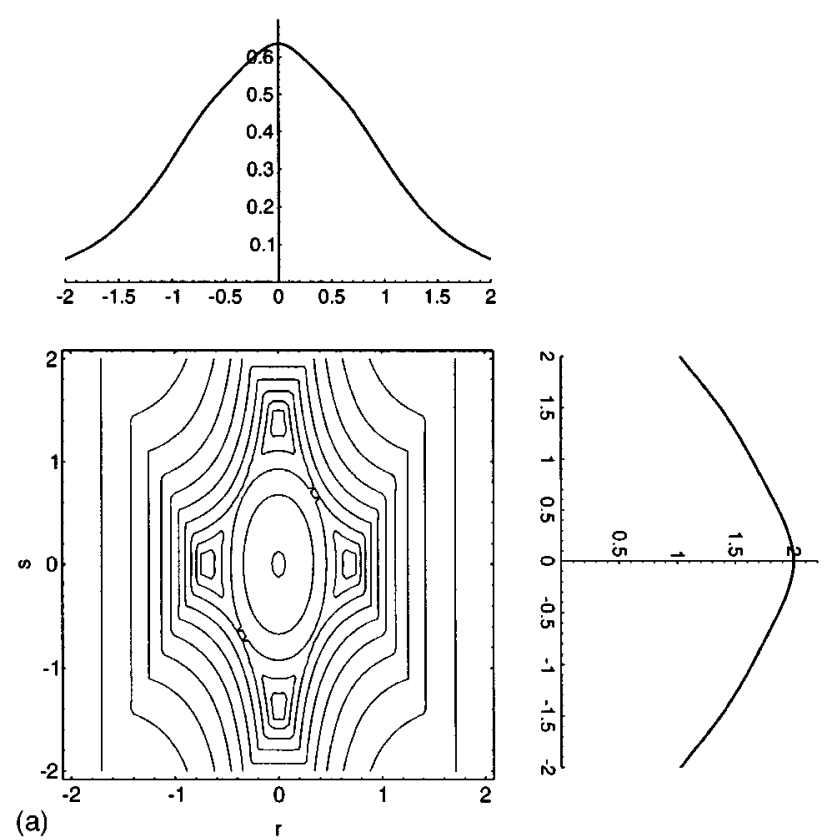

(a)
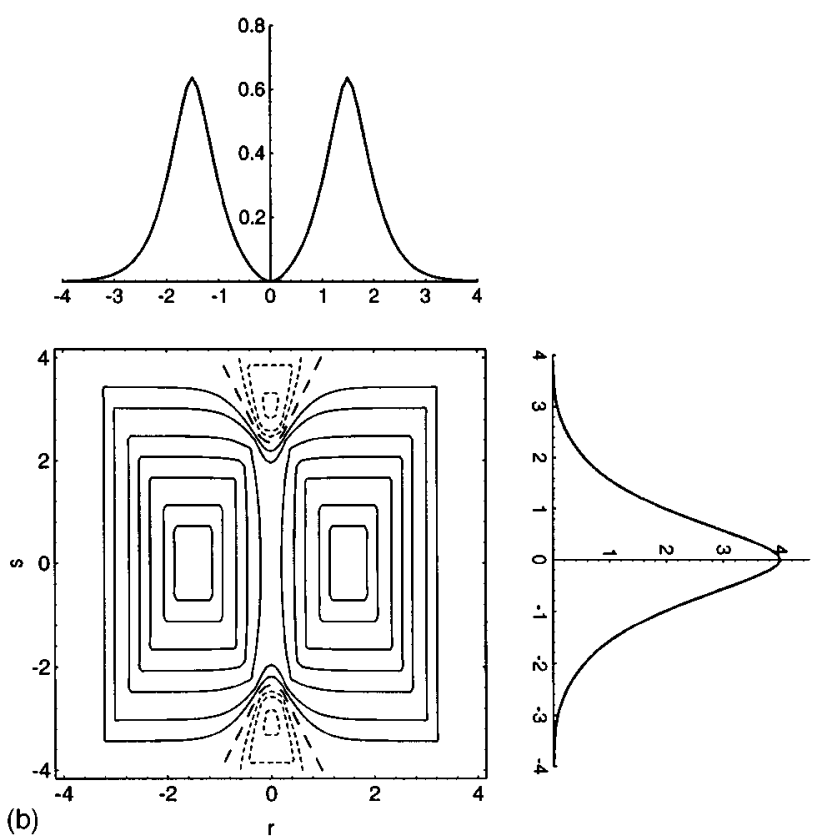

(b)

FIG. 1. Schematic representation of the one-particle reduced density matrix (contour plot), the reciprocal form factor (right-hand curve) and the parity function (top curve) of a one-dimensional "molecular hydrogen" system (a) and a one-dimensional "helium atom pair" system (b) (see the text for details). The contour-lines are $0.1 \ldots(0.1) \ldots 1.0$ for (a) and $\{0, \pm 0.01, \pm 0.02, \pm 0.05,0.1,0.2,0.5,1.0\}$ for (b).

\section{TWO EXAMPLES}

To demonstrate the information contained in the two projections of the density matrix, we first choose two neartrivial model systems (Fig. 1). The one is a one-dimensional model of the $\mathrm{H}_{2}$ molecule (a), the other a similar model of a pair of helium atoms (b).

In the first case, our model wave function is a $2 \times 2$ Slater determinant in a minimal basis of Slater orbitals in one dimension; (its general arguments will be labeled $x$ in the following; internal and external coordinates are the scalars $r$ and $s$ in this section). Therefore, the only occupied orbital to be considered is

$$
\begin{aligned}
& \phi_{s}(x)=(2+2 S)^{-1 / 2}(\phi(x+R / 2)+\phi(x-R / 2)), \\
& \phi(x)=\sqrt{\xi} \mathrm{e}^{-\xi|x|} .
\end{aligned}
$$

Here, $R$ is the distance between one center and the other, and the exponent $\xi$ describes the spatial extent of the primitive basis functions $\phi(x)$. The subscript $s$ denotes "symmetric," and it labels quantities pertaining to the "molecular" orbital, whereas quantities without label are meant to be "atomic," i.e., deriving from the primitive basis function. The function $S$ is the overlap integral between the basis functions and occurs in the normalization factor. The density matrix for this primitive system may be readily written down in $(r, s)$ coordinates

$$
\begin{aligned}
\widetilde{\rho}_{s}(r, s)= & 2(2+2 S)^{-1}(\widetilde{\rho}(r+R / 2, s)+\widetilde{\rho}(r-R / 2, s) \\
& +\widetilde{\rho}(r, s+R)+\widetilde{\rho}(r, s-R)) \\
\widetilde{\rho}(r, s)= & \xi \mathrm{e}^{-\xi(|r+s / 2|+|r-s / 2|)} .
\end{aligned}
$$

The contribution $\widetilde{\rho}(r, s)$ arises from the primitive. Integration over $r$ and $s$ yields the desired reciprocal form factor $B_{s}(s)$ and the parity function $P_{s}(r)$, respectively;

$$
\begin{aligned}
B_{s}(s) & =2(2+2 S)^{-1}(2 B(s)+B(s+R)+B(s-R)), \\
P_{s}(r) & =2(2+2 S)^{-1}(P(r+R / 2)+P(r-R / 2)+2 P(r)) \\
& =\pi^{-1} B_{s}(2 r),
\end{aligned}
$$

in terms of the primitive contributions

$$
\begin{aligned}
& B(s)=(1+\xi|s|) \mathrm{e}^{-\xi|s|} \\
& P(r)=\pi^{-1}(1+2 \xi|r|) \mathrm{e}^{-2 \xi|r|}=\pi^{-1} B(2 r) .
\end{aligned}
$$

It may be noted that for purely inversion symmetric functions, the relation $P(r)=B(2 r) / \pi$ always holds. As a result, the parity function $P_{s}(r)$ is (in this simple example) just a rescaled version of the reciprocal form factor $B_{s}(s)$ (see also Ref. 31). The shape of these functions is displayed in Fig. 1a. The center of the figure shows a contour-plot of the SPDM $\tilde{\rho}_{s}(r, s)$ of this system. One consequence of the independentparticle model adopted here is the equality of the peak heights in the diagonal ( $s=0$; density) and the non-diagonal $(s \neq 0$; overlap) regions of the density matrix. The reciprocal form factor (plot on the right) registers the bonding nondiagonal regions in the wings as slight shoulders. The local parts of the SPDM contribute to this function only at $s=0$, i.e., in the form of the normalization. In contrast to this, the parity function $P(r)$ (plot on the top) is dominated near the bond-midpoint by these non-diagonal regions, and it shows the nuclei as shoulders in the wings. It is therefore suitable for assigning the characteristic nonlocal contributions of the SPDM to the region between bound centers.

For the corresponding antisymmetric function

$$
\phi_{a}(x)=(2-2 S)^{-1 / 2}(\phi(x+R / 2)-\phi(x-R / 2)),
$$


the treatment is completely analogous, leading to $P_{a}(r)=-B_{a}(2 r) / \pi$ and a correspondingly different reciprocal form factor;

$$
B_{a}(s)=(2-2 S)^{-1}(2 B(s)-B(s+R)-B(s-R)) .
$$

Since this does not introduce anything significantly new, these functions are not displayed. However, for the case of a one-dimensional "helium pair" (Fig. 1b), where both the symmetric and antisymmetric functions above are doubly occupied, no trivial scaling relation exists between the parity function $P$ and the reciprocal form factor $B$. In this case one obtains

$$
\begin{aligned}
\widetilde{\rho}_{m}(r, s)=2\left(1-S^{2}\right)^{-1}[\widetilde{\rho}(r+R / 2, s)+\widetilde{\rho}(r-R / 2, s) \\
-S\{\widetilde{\rho}(r, s+R)+\widetilde{\rho}(r, s-R)\}], \\
B_{m}(s)=2\left(1-S^{2}\right)^{-1}[2 B(s)-S\{B(s+R)+B(s-R)\}], \\
P_{m}(r)=2\left(1-S^{2}\right)^{-1}[P(r+R / 2)+P(r-R / 2)-2 S P(r)],
\end{aligned}
$$

again in terms of primitive contributions. The subscript $m$ stands for "mixed." Note that for $s=0$, the reciprocal form factor $B_{m}(s)$ attains a value of 4 , the number of particles in this model system, owing to the fact that $S=B(R)$. However, the parity function $P_{m}(r)$ becomes zero at $r=0$, expressing the fact that there is a symmetric and an antisymmetric function with equal occupation. The above functions for the mixed system are displayed in Fig. 1b. The features of the SPDM (central contour plot) are in some way complementary to the ones in Fig. 1a. The non-diagonal regions exhibit negative areas around $s= \pm R$, owing to a predominance of negative phase-relations between the two centers. The impact of these regions on the reciprocal form factor $B_{m}(s)$ (right-hand-side plot) is the lowering of the value at this range, i.e., a narrowing of the function. The effect on the parity function $P_{m}(r)$ is more dramatic; the negative regions cancel on integration with the positive contributions from the charge density between the centers, to give exactly zero for the independent-particle model. Instead of a shared positive area for the bonding case, two clearly separated ones arise for the closed-shell interaction case.

From an extension of the above considerations for primitive models, it is possible to extract a good deal of information about the bonding in molecular systems from the parity function $P(\vec{r})$. Examples for this are given in the following section.

\section{PARITY FUNCTIONS FOR SOME MOLECULES}

The simplest examples for bonding and non-bonding molecular interactions are given by the hydrogen molecule and the dihelium atom pair. Primitive models of those systems were shown in the previous section, and the extension to three dimensions does not change the qualitative features. Therefore, no contour plots of those systems are shown here.

All wave functions employed in the following are of the RHF type, calculated with a valence triple-zeta basis set with polarization $\left(6-311 \mathrm{G}^{* *}\right.$ in "Gaussian" notation $\left.{ }^{33}\right)$. The
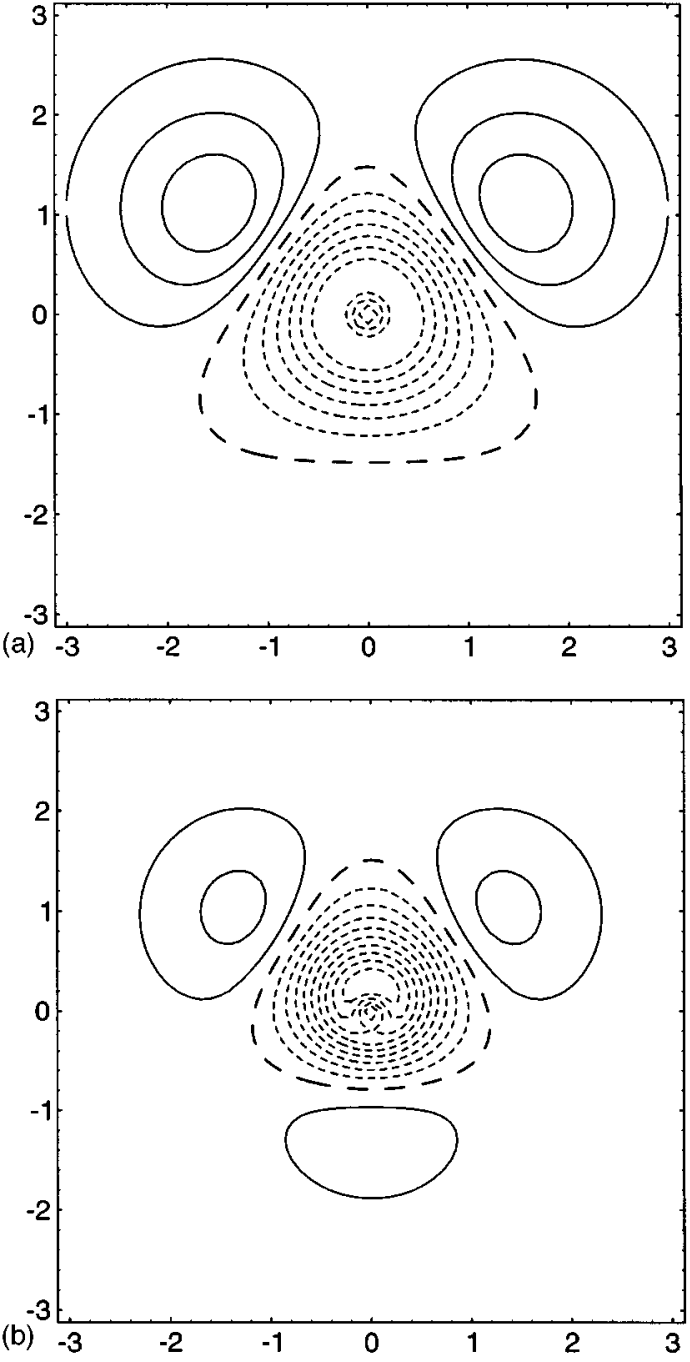

FIG. 2. Sections through the parity functions of methane (a) and water (b). The displayed plane is the molecular symmetry plane containing three atoms. The contour-line distance is 0.01. Negative contours are displayed dotted, positive ones are full, the zero contour is dashed. All scales are in atomic units, i.e., length $a_{0}$ and parity function $e / \hbar^{3}$.

quantity $P(\vec{r})$ cannot be expected to be very sensitive to basis-set effects, since it maps out qualitative bond features. However, there is an obvious impact of electron correlation on the grounds that the parity function depends in a differential manner on the occupation numbers of the SPDM. In some cases, contributions of different orbitals cancel exactly in the RHF approximation, and they do not for correlated wave functions. To obtain a basic understanding of the patterns occurring for given bond types, it is nevertheless preferable to restrict oneself to single-determinant representations.

We start with simple first-row hydrides (Fig. 2), namely the methane (a) and the water (b) molecules. The essential bond type in those may be denoted $(s-p) \sigma$. The oneparticle density matrix of such a system exhibits a nodal structure in the off-diagonal region, mapping the phasechange of the $p$-orbitals on the central atom (see Ref. 14 for 
details). This structure is retained in the parity function in the form of a nodal surface, intersecting the bond-axis almost perpendicularly. For low momenta, the Wigner function of molecules exhibits large negative regions in the vicinity of first-row atoms with $p$-occupation (see Refs. 30, 34). It is therefore a general feature of the parity function to have negative basins around those atoms whenever the molecule fulfills the octet rule. The latter supplies the atoms with a shell structure similar to that of neon, whose Wigner function at the origin is $-2 / \pi^{3}$ in the Hartree-Fock approximation. On the other hand, the generally positive parity function around hydrogen centers (see Refs. 29, 34) forces a nodal plane on the bonds. This plane has to be closed, since it is clear that $\lim _{r \rightarrow \infty} P(\vec{r})>0$. The qualitative arguments made here are essentially of an atomic nature; bond types and the resulting topological features in the parity function have the character of a refined "balls-and-sticks" model that is reminiscent of the VSEPR model ${ }^{35}$ and its relatives. There are great similarities between the functions for methane (a) and water (b). In the latter case, the region behind the nucleus that is the location of the free electron pairs features another slight maximum. This ability of the parity function, to resolve areas of electron accumulation to some degree, is commonly observed. In the case of methane, the corresponding feature is a saddle point arising from the out-of-plane hydrogen centers.

The standard bond in organic molecules is, of course, the $\mathrm{C}-\mathrm{C}$ single bond. It is usually considered to be of the $(p-p) \sigma$ type, and the off-diagonal structure of the SPDM consequently exhibits two intersecting nodal surfaces. ${ }^{14}$ The projection of the positive and negative regions onto $P(\vec{r})$ places both of those on the bond-axis, thus separating two negative carbon atomic regions from a positive bond or overlap region. The examples shown in this paper are two conformations of ethane, staggered and eclipsed (Fig. 3). The staggered form (a) has an inversion center at the $\mathrm{C}-\mathrm{C}$ bond midpoint, and since there is one more symmetric orbital than antisymmetric ones, the value at that point is $2 / \pi^{3}$ in the independent-particle model. Although this is not the case for the eclipsed form (b), the value at the $\mathrm{C}-\mathrm{C}$ midpoint is almost as large. It may be concluded that, although there is an influence of the symmetry of the nuclear framework on the parity function, its qualitative features are more strongly dependent on the electronic structure.

Multiple bonds contribute negatively to the parity function in the bond region close to the axis, and positively away from it. This is easily understood if one recalls the ungerade nature of $\pi$-type orbitals, and if one takes into account that away from the bond axis, these contributions are similar to $\sigma$-bonds off-axis (the "banana-bond" picture). As a result, the parity function of multiply bonded systems features a dramatic separation of $\sigma$ and $\pi$ regions. The standard example for a double-bond is ethylene (Fig. 4), a molecule with an inversion center. One consequence of this high symmetry is the occurrence of a degenerate point in the parity function: since there are just as many gerade as ungerade orbitals occupied, the value at the center of symmetry is exactly zero
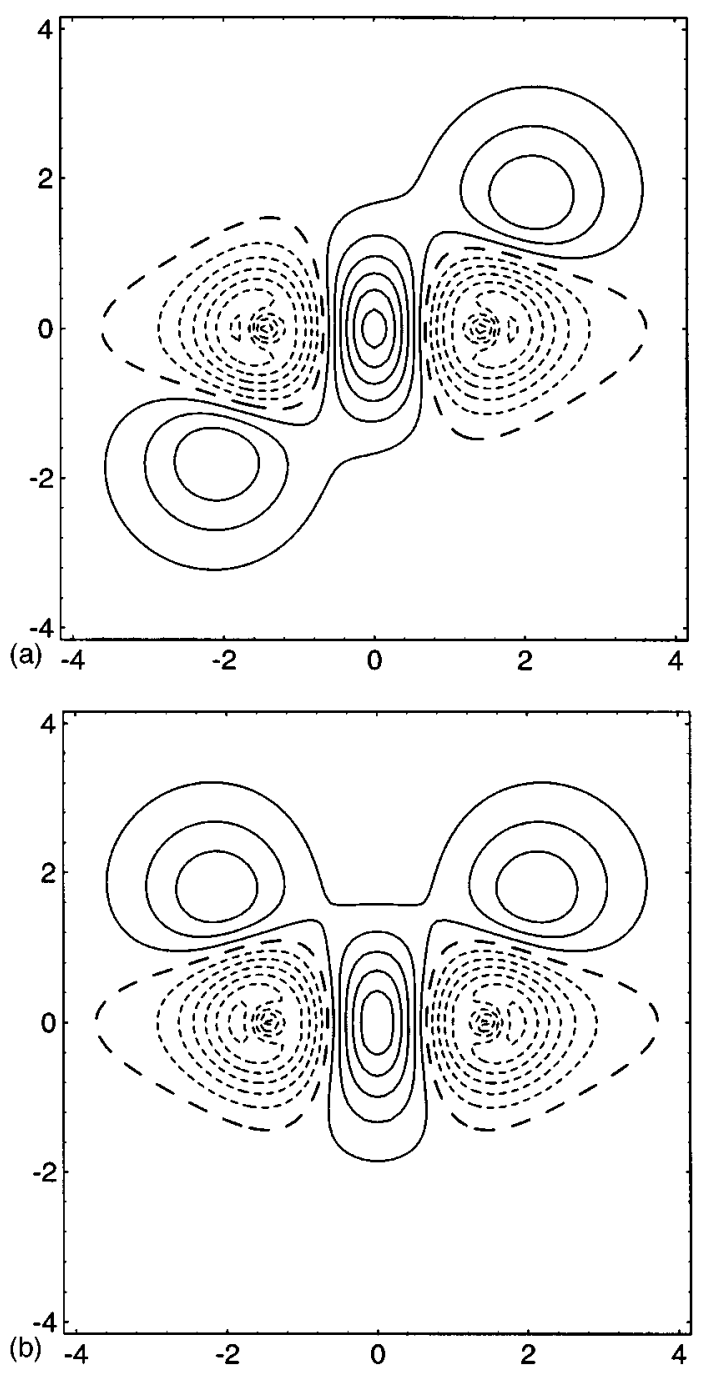

FIG. 3. Sections through the parity function of ethane in the staggered (a) and eclipsed (b) conformation. The $\sigma_{v}$ mirror plane is displayed in both cases. Contour lines are as in Fig. 2.

in the Hartree-Fock approximation. This is a similar situation as in symmetric closed-shell systems (such as pairs of noblegas atoms). However, in the latter cases there are no pronounced off-axis regions in $P(\vec{r})$, which is in contrast to the situation here, where marked positive features are observed. The cancellation of symmetric and antisymmetric contributions happens between bonding $\sigma$ and $\pi$ functions for the double bonds, and between bonding and antibonding functions (of either type) for the closed-shell cases (see also Fig. $7 \mathrm{~b}$ for the case of $\mathrm{LiF}$ ).

An isoelectronic system with a lower symmetry is formaldehyde (not shown), where the principal features of the parity function are very similar to what we observe in $\mathrm{C}_{2} \mathrm{H}_{4}$. No degenerate point is present, instead a slightly negative area occurs on the bond axis. The nodal lines close in-plane and are deformed in direction of the oxygen atom. But the characteristic positive $\pi$-regions are there. It is also remarkable that the lone electron pairs on the oxygen atom 

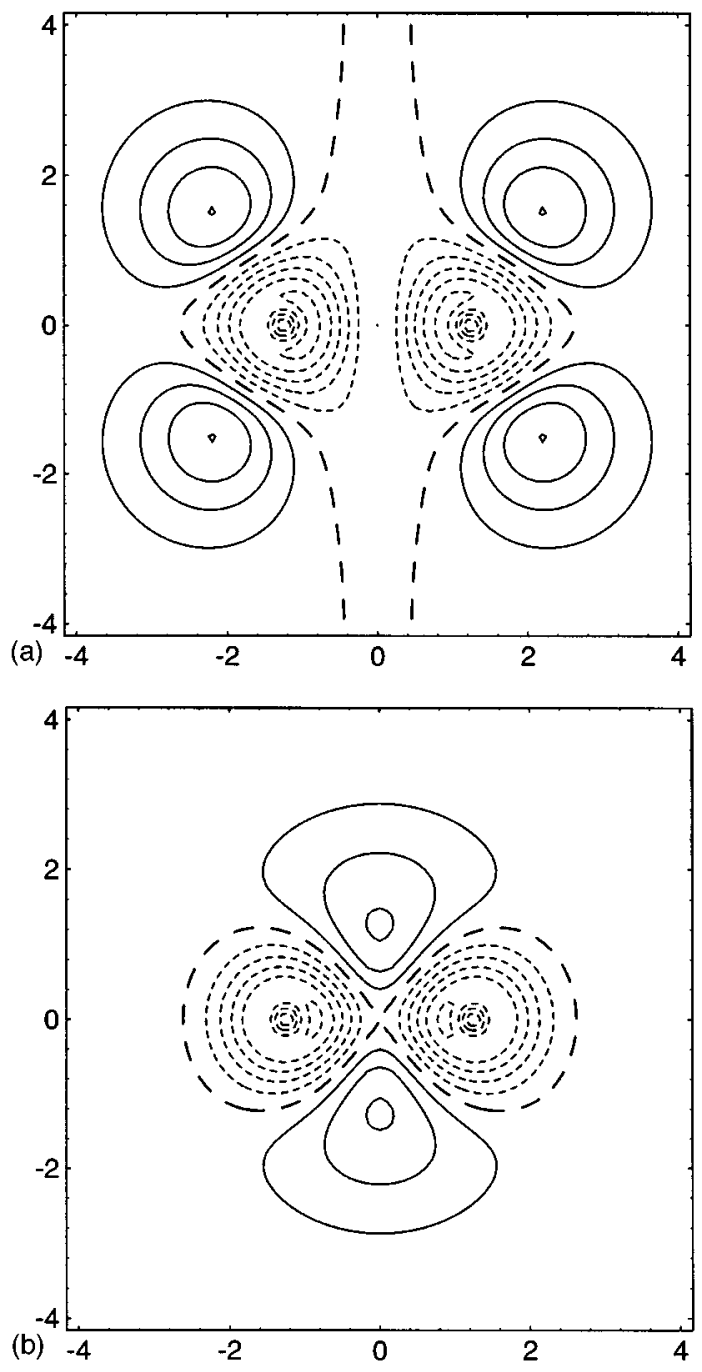

FIG. 4. Sections through the parity function of ethylene in the molecular plane (a) and perpendicular to it (b). Contour lines are as in Fig. 2.

lead to a positive region behind that center, corresponding to the more marked features arising from the hydrogen atoms in ethylene.

It might be expected to find a mixture of the features for $\mathrm{C}-\mathrm{C}$ single and double bonds in the case of aromatic systems. The standard example of benzene confirms this (Fig. 5). While one finds positive values of $P(\vec{r})$ at the bond midpoint (i.e., like in ethane, but lower), there are also $\pi$ contributions visible parallel to the molecular plane, but off-axis (see Fig. 5b). They would not be as prominent if the bond in benzene were single, and there would be much lower or even negative values on the axis, if it were double. The author is given to speculations about the inference of bond orders from these values without reference to basis functions. However, it is unclear how the cases of bonding contributions from $\pi$ orbitals should be distinguished from antibonding ones from $\sigma^{*}$, if only a single value at, e.g., the bond midpoint is taken into account. However, even without an obvious scheme for bond orders, it is gratifying to observe the
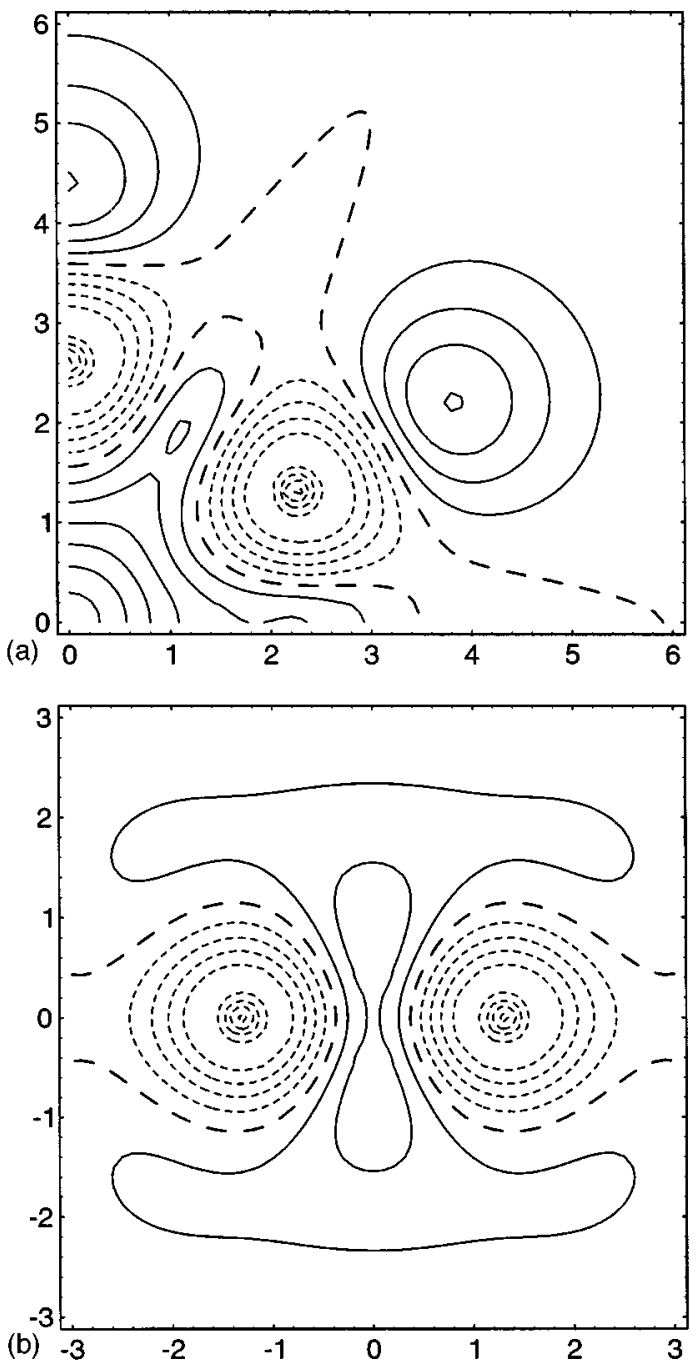

FIG. 5. Sections through the parity function of benzene in the molecular plane (a) (only one quadrant) and perpendicular to it, including a $\mathrm{C}-\mathrm{C}$ bond (b). Contour lines are as in Fig. 2.

extent to which the parity function includes information about the type of bonding present in a molecule.

If the contributions from the $\pi$ system in a molecule become even stronger, as is the case for triple bonds (Fig. 6), the character of the bond region is essentially antisymmetric, and the parity function $P(\vec{r})$ assumes negative values on the axis. The classical examples are nitrogen (a) and acetylene (b). The cross features of these two systems are remarkably similar. They are dominated by a merging of the negative regions surrounding the atoms. Positive areas behind them arise from bonds to hydrogen (b) or from lone pairs (a). Again, the equivalence of these two elements is apparent, just as in the methane/water (Fig. 2) and the ethylene/ formaldehyde (Fig. 4) cases. A positive structure that is dominated by the $\pi$-system is placed cylindrically around the central negativity region. In the case of $\mathrm{N}_{2}$ (a), there is a tendency of this feature to merge with the free-pair region behind the nuclei, but it is still pronounced enough to form a distinguished ring. One may argue that the radius at which 

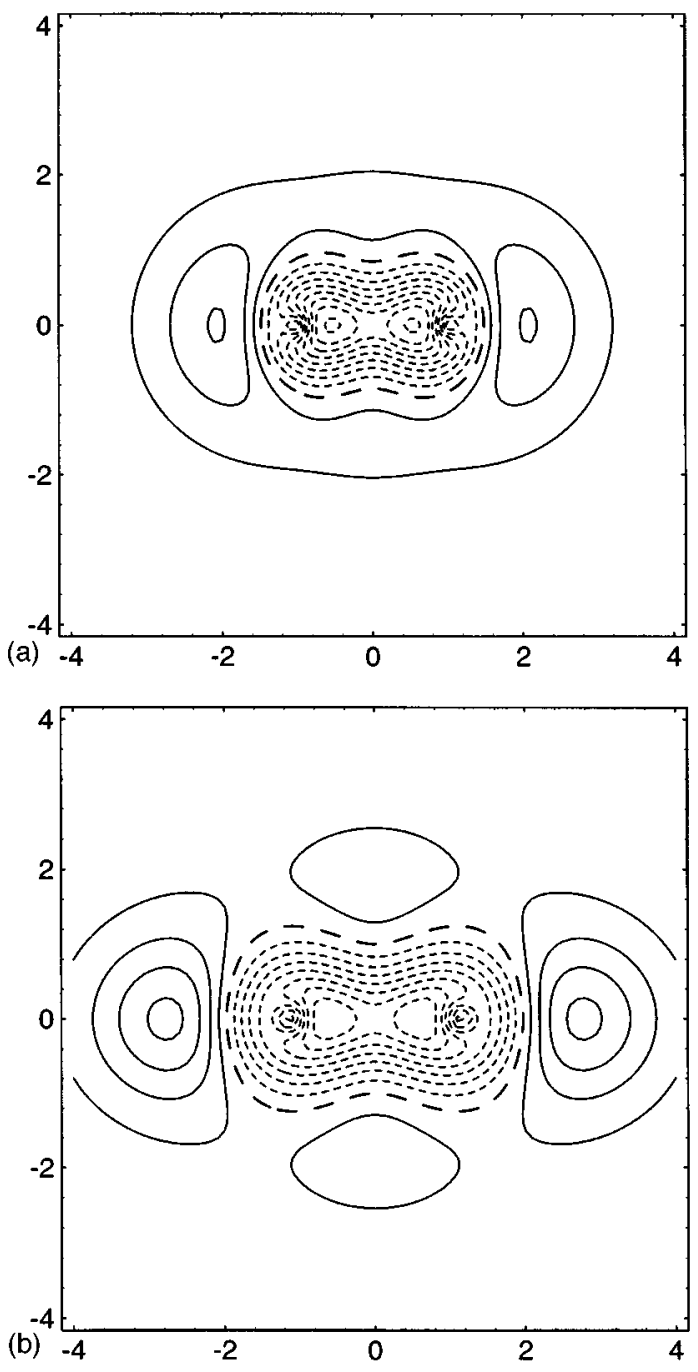

FIG. 6. Sections through the parity functions of nitrogen (a) and acetylene (b). The planes displayed include the molecular axes. Contour lines are as in Fig. 2.

this ring occurs is the characteristic one for the $\pi$-system since there, the net overlap is maximal.

The next two examples concern partly ionic bonds. Since these are closed-shell interactions, the adhesive forces are of an electrostatic origin rather than interference effects (as in covalent bonds). Therefore, the off-diagonal regions of the one-particle density matrix are considerably weaker than in covalently bonded systems, and for systems with clearly ionic character, they are even antibonding in their topology (see Ref. 14). This has a severe impact on the parity function. Figure 7 shows the cases of lithium hydride (a) and lithium fluoride (b). The LiH system (a) is isoelectronic to the helium dimmer. It is not completely ionic, but has considerable covalent contributions (a fact that has been clearly demonstrated by employing projected pair densities ${ }^{20}$ ). As a result, some positive contributions occur in the bond region, but they are weak. A clear separation of two subsystems is observed, and the function takes its lowest value between the
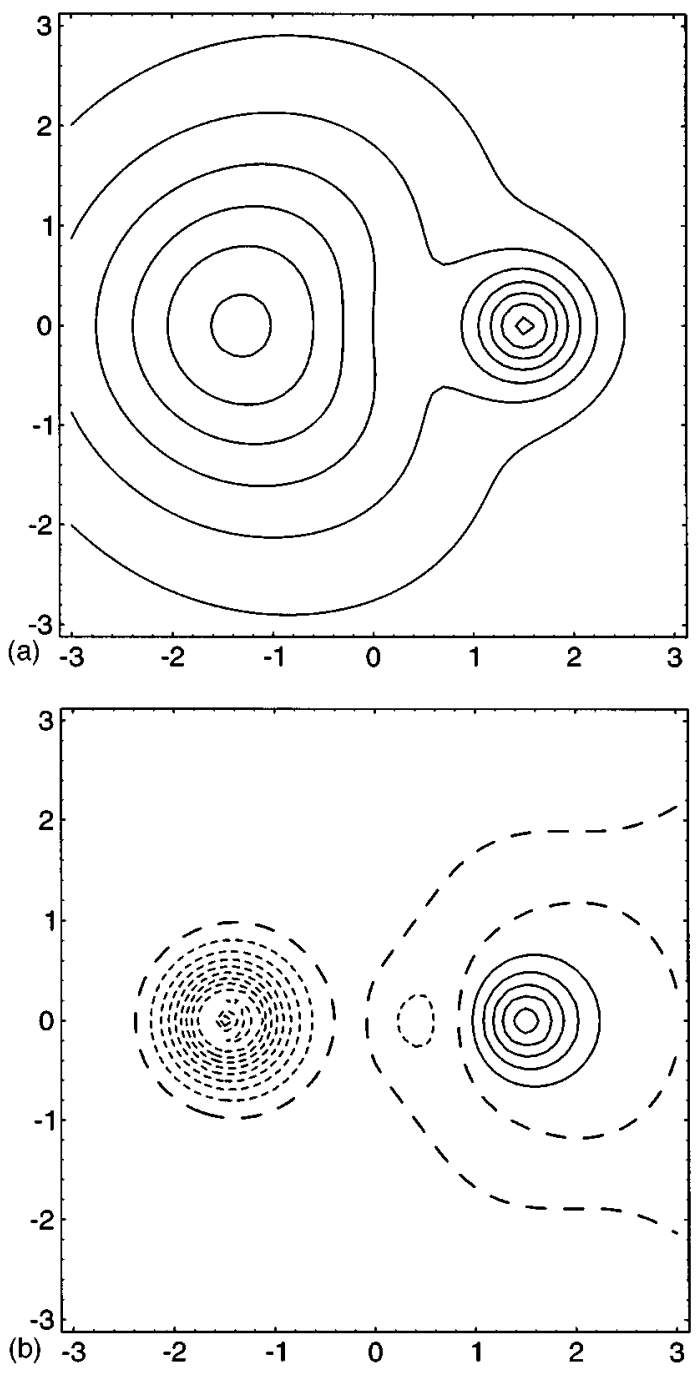

FIG. 7. Sections through the parity functions of lithium hydride (a) and lithium fluoride (b). The planes displayed include the molecular axes. The lithium centers are to the right. Contour lines are as in Fig. 2.

centers, as opposed to a covalent $(s-s) \sigma$ structure, where the bond region shows a maximum.

A more obvious example for a closed-shell ion pair is lithium fluoride (b). The structure of the parity function of this system is more reminiscent of an atom pair than of a true molecule (as treated above). In the bond region, the values are very low and slightly negative. This is a consequence of the cancellation of contributions from bonding and antibonding $\sigma$-orbitals. The presence of the nodal lines arises from the $(s-p)$-nature of those orbitals. Both examples in Fig. 7 confirm the rule that low values of $P(\vec{r})$ in a "flat" area between the bound centers indicate a closed-shell type of interaction. The separation is reflected more strongly in the parity function than in other distributions such as charge or momentum densities.

The above molecules exhibit simple standard types of bonding. We close this section by treating two examples where the situation is less clear-cut (Fig. 8). The first is the three-center bonds in diborane (a); we depict the plane that 

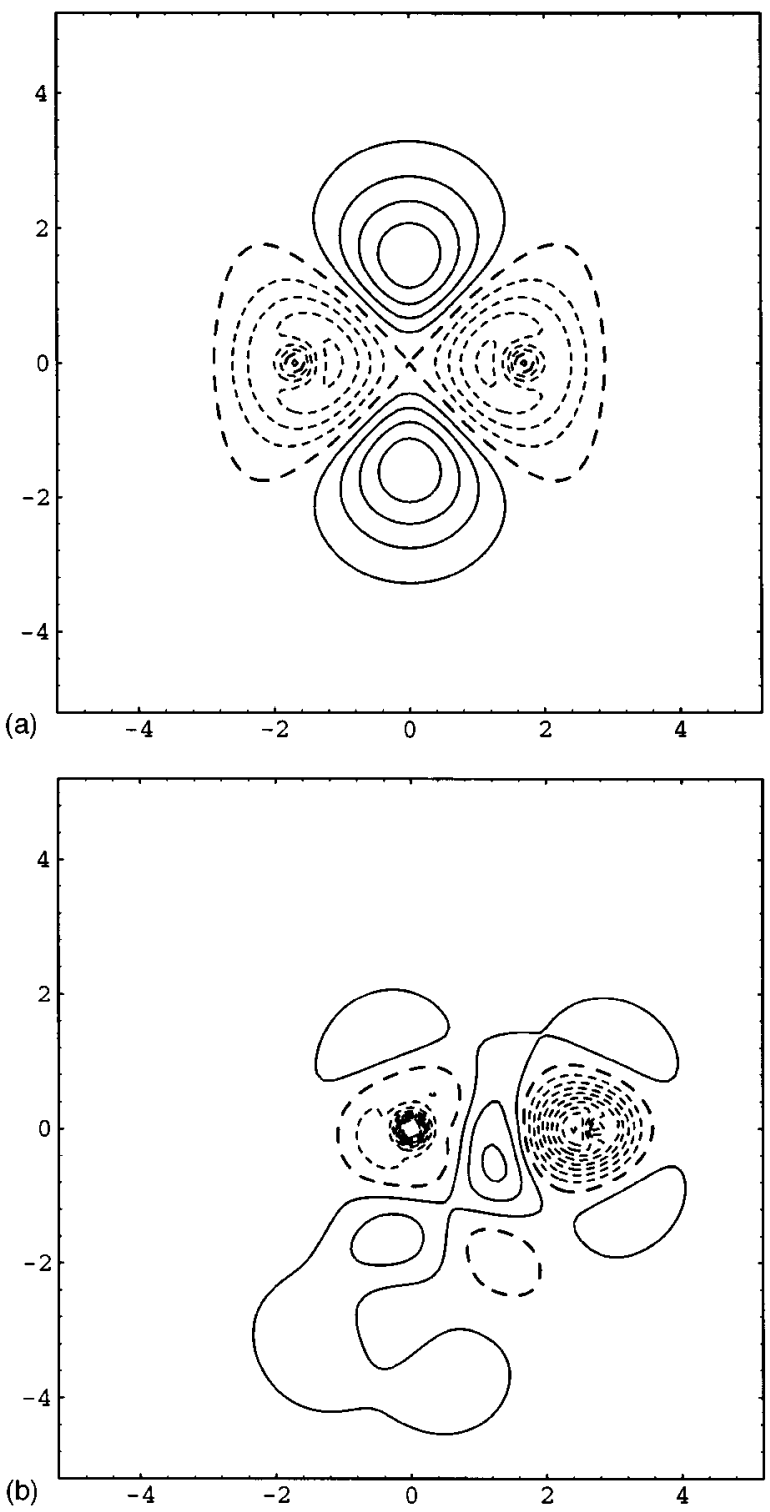

FIG. 8. Sections through the parity functions of diborane (a) and chloric acid (b). For (a), the plane displayed includes the boron centers and the bridging hydrogen nuclei. For (b), it is perpendicular to the $\mathrm{O}-\mathrm{Cl}-\mathrm{O}$ plane and includes one $\mathrm{Cl}-\mathrm{O}$ bond. The $\mathrm{Cl}$ atom is at the origin, and the $\mathrm{x}$-axis coincides with the bond. Contour lines are as in Fig. 2.

includes the two boron and the bridging hydrogen atoms. It is obvious that the situation in the bonding region is completely equivalent to the one observed in ethylene (Fig. 4b), i.e., that from the electronic point of view, the BHB-threecenter bonds act like a double bond between the boron centers. The hydrogen nuclei are submerged in a region of maximal overlap, and modify the picture just by leading to a relative increase in the parity function off the symmetry axis. This suggests a model of the three-center bonds, in which electrons are "borrowed" from hydrogen to fulfill the octet rules on the boron atoms.

The last example is a so-called hypervalent molecule, namely chloric acid $\mathrm{HOClO}_{2}$ (Fig. 8b). ${ }^{36}$ Structure formulae that assume double bonds between chlorine and oxygen lead to a violation of the octet rule on chlorine. If the latter is assumed to be fulfilled, formal charges must be assigned to the centers. It is therefore likely that neither double nor single-bond descriptions are completely valid. Fig. 8b shows a section through $P(\vec{r})$ of this molecule, where the central chlorine $($ at $\mathrm{x}=\mathrm{y}=0)$ and one "doubly bonded" oxygen atom (on the positive x-axis) are included. We note a peaking of the parity function near the center of bond that is reminiscent of $(p-p) \sigma$ single bonds encountered earlier for ethane (Fig. 3). However, somewhat enhanced off-axis contributions are visible as well, and the maximum is comparably low and shifted off-axis. This is the consequence of the partial $\pi$-character of the $\mathrm{Cl}-\mathrm{O}$ bond. The bond overlap region merges with the characteristic free-electron-pair regions behind the chlorine and oxygen centers. From this description, one would characterize this bond as single, but with some contributions from the $\pi$-system. Of course, its partial double-bond character is already expressed in the short bond length $(1.44 \AA$, as compared to $1.71 \AA$ for the " $\mathrm{Cl}-\mathrm{O}$ single bond" in the same molecule). A Mulliken population analysis confirms that $\mathrm{Cl}$ carries considerable positive charge $(+1.5)$, and the Mayer bond $\operatorname{order}^{37}$ for the $\mathrm{Cl}-\mathrm{O}$ is 1.36 , i.e., closer to single than to double. Note that in Fig. 8b, the features on the lower part of the plot are due to the $\mathrm{Cl}-\mathrm{O}-\mathrm{H}$ fragment which is out of plane.

\section{SUMMARY}

It has been deemed beneficial to extend the analysis of molecular electronic structure from the familiar charge density to nonlocal regions of the single-particle reduced density matrix, if a more complete picture is desired. Difficulties arising from the multidimensional nature of the density matrix may be overcome by either restricting the analysis to sections and selected directions, or by projecting the quantity onto subspaces of interest. Two natural choices of such subspaces are discussed in this paper. The one spanned by the internal coordinate is characterized by the reciprocal form factor $B(\vec{s})$, a quantity that has found widespread applications in the past, and is closely related to the electronic charge density in momentum space. The other, spanned by the complimentary external coordinate may be described in terms of a parity function $P(\vec{r})$, which is introduced in this paper. The latter bears a close relation to the phase-space representation of the density matrix, more specifically, it is an initial value of the Wigner function.

Some basic properties of this function were discussed here with the examples of primitive one-dimensional systems. It was demonstrated that the presence (or absence) of covalent chemical bonds in a system influence the topology of this function in a profound manner, and that it is therefore possible to link qualitative features of the function to certain "bond types." More specifically, these links are:

- A covalent $\sigma$ bond in which the main atomic components are of $s$-type, leads to maxima located on the bond between the bound centers. If the atomic components are mainly of $p$-type, this maximum still occurs, but is separated from the centers by nodal planes that 
extend approximately perpendicular off the bond axis. Mixed atomic contributions are characterized by the presence of one nodal plane in the vicinity of the bond midpoint.

- Double bonds exhibit comparably small, sometimes negative values of the parity function on the bond axis, and positive regions away from it. These positive values are located in the $\pi$-region. The separation of $\sigma$ and $\pi$ regions in multiply bound systems is very marked in the parity function, and may be used to define a characteristic distance.

- Triple bonds lead to relatively strong negative areas close to the bond axis, with positive contributions in the $\pi$ region. The latter are weaker than in the case of double bonds, since they are tangentially delocalized.

- Non-bonding electron pairs in many cases lead to features reminiscent of $\sigma$ bonds. However, they are weaker and less clearly defined.

- For aromatic systems, a mixture of features from double and single bonds may be expected. A clear definition of the $\pi$-system is observed and an orbitallike separation of $\pi$ and $\sigma$ parts is possible.

- Systems in which the bond is mainly of an ionic or van der Waals type show a marked separation of the bound centers, with rather low values of the parity function in the bond region. Occasionally occurring nodal planes are influenced by the type of the main atomic contributions from the centers.

- For less clear-cut examples, it is sometimes helpful to draw apologies to a known type to interpret the topology of the parity function. Mixed types may be derived by interpolation.

It is, of course, not always possible to distinguish clearly between types, particularly without prior knowledge of the situation. However, the parity function introduced here offers a rather pictorial "fingerprint" of the off-diagonal regions of the density matrix, and is therefore perfectly suited to supplement the charge density as a means of characterizing the SPDM in position space. It has the additional advantage that it does not give up the local character of the charge density completely, i.e., that it is (unlike the reciprocal form factor) still a function of the absolute position. On the downside, it includes almost no information about the kinematic state of the electrons in the system. For the analysis of bond situations, it is preferable to an orbital-by-orbital description, since it does not suffer from the ambiguities of the latter, and it also tends to localize the main features onto the bond region. For larger systems, many orbitals would have to be taken into account, and the parity function offers a meaningful way of condensing the inherent information. We hope that it proves useful as a tool for understanding and explaining electronic structure beyond the analysis of charge distributions.

\section{ACKNOWLEDGMENTS}

The Danish Natural Science Research Council supplied financial support for the work that is reported in this paper. I would like to thank Professor Vedene H. Smith, Jr. and Professor Jens-Peder Dahl for reading the manuscript and making suggestions.

${ }^{1}$ P. A. M. Dirac, Proc. Cambridge Philos. Soc. 26, 376 (1930).

${ }^{2}$ K. Husimi, Proc. Phys. Math. Soc. Jpn 22, 264 (1940).

${ }^{3}$ P. O. Löwdin, Phys. Rev. 97, 1474, 1490, 1509 (1955).

${ }^{4}$ R. McWeeny, Proc. R. Soc. London, Ser. A 232, 114 (1955).

${ }^{5}$ R. McWeeny, Rev. Mod. Phys. 32, 335 (1960).

${ }^{6}$ C. A. Coulson and H. C. Longuet-Higgins, Proc. R. Soc. London, Ser. A 191, 39 (1947); ibid. 193, 447, 456 (1948).

${ }^{7} \mathrm{~W}$. Weyrich, Einige Beiträge zur Comptonspektroskopie (Technische Hochschule Darmstadt, Darmstadt, 1978), Habilitationsschrift.

${ }^{8}$ W. Weyrich, 1985, lecture given on the Sagamore VIII Conference on Charge, Spin and Momentum Densities in Sånga Säby, Sweden.

${ }^{9}$ R. Benesch, S. R. Singh, and V. H. Smith, Jr., Chem. Phys. Lett. 10, 151 (1971)

${ }^{10}$ F. M. Mueller, Bull. Am. Phys. Soc. 19, 200 (1974).

${ }^{11}$ B. G. Williams, Compton Scattering (McGraw-Hill, London, 1977).

${ }^{12}$ G. Sperber, Int. J. Quantum Chem. 5, 189 (1971).

${ }^{13}$ S. R. Gadre, S. A. Kulkarni, and R. K. Pathak, Phys. Rev. A 40, 4224 (1989).

${ }^{14}$ H. Schmider, K. E. Edgecombe, V. H. Smith, Jr., and W. Weyrich, J. Chem. Phys. 96, 8411 (1992).

${ }^{15}$ T. Asthalter and W. Weyrich, Ber. Bunsenges. Phys. Chem. 96, 1747 (1992).

${ }^{16}$ H. Schmider and V. H. Smith, Jr., Theoret. Chim. Acta 86, 115 (1993).

${ }^{17}$ C. A. Coulson, Proc. Cambridge Philos. Soc. 37, 55 (1941); also ibid. 37, 74 (1941); C. A. Coulson and W. E. Duncanson, ibid. 37, 67 (1941); W. E. Duncanson, ibid. 37, 97 (1941); ibid. 39, 180 (1943); W. E. Duncanson and C. A. Coulson, ibid. 37, 406 (1941); ibid. 38, 100 (1942).

${ }^{18}$ A. J. Thakkar, A. C. Tanner, and V. H. Smith, Jr., in Density Matrices and Density Functionals, edited by R. Erdahl and V. H. Smith, Jr. (Reidel, Dordrecht, 1987), pp. 327-337.

${ }^{19}$ A. J. Thakkar, in Density Matrices and Density Functionals, edited by R. Erdahl and V. H. Smith, Jr. (Reidel, Dordrecht, 1987), pp. 553-581.

${ }^{20}$ J. Wang and V. H. Smith, Jr., Int. J. Quantum Chem. 49, 147 (1994).

${ }^{21}$ M. Weisser, Ph.D. thesis, Universität Konstanz, 1996, also references therein.

${ }^{22} \mathrm{H}$. Weyl, The Theory of Groups and Quantum Mechanics (Dover, New York, 1931).

${ }^{23}$ E. Wigner, Phys. Rev. A 40, 749 (1932).

${ }^{24}$ M. Hillery, R. F. O'Connell, M. O. Scully, and E. P. Wigner, Phys. Rep. 106, 121 (1984).

${ }^{25}$ A. Royer, Phys. Rev. A 15, 449 (1977).

${ }^{26}$ A. Royer, Phys. Rev. Lett. 55, 2745 (1985).

${ }^{27}$ A. Grossmann, Commun. Math. Phys. 48, 191 (1976).

${ }^{28}$ J. P. Dahl, Physica A 112, 439 (1982).

${ }^{29}$ J. P. Dahl and M. Springborg, Mol. Phys. 47, 1001 (1982).

${ }^{30}$ M. Springborg and J. P. Dahl, Phys. Rev. A 36, 1050 (1987).

${ }^{31}$ R. A. Bonham and W. Weyrich, J. Braz. Chem. Soc. 1, 7 (1990).

${ }^{32}$ One may argue that at least the value of the momentum density at the origin is retained and spatially resolved. It is not clear to the author how this fact pertains to the idea of "low-momentum electrons," a subject to which he has devoted another paper, employing a related, the so-called Husimi distribution [see Schmider, J. Chem. Phys. 105, 3627 (1996)]. Incidentally, the Fourier transform of the parity function $P(\vec{r})$ is a section through the SPDM in momentum space, which covers the non-local regions in that representation and passes through the origin of $p$-space. It is therefore complementary to the momentum density.

${ }^{33}$ R. Krishnan, J. S. Binkley, R. Seeger, and J. A. Pople, J. Chem. Phys. 72, 650 (1980).

${ }^{34}$ H. Schmider and J. P. Dahl, Int. J. Quantum Chem. 60, 439 (1996).

${ }^{35}$ R. J. Gillespie, Molecular Geometry (Van Nostrand Reinhold, London, 1972).

${ }^{36}$ For the $\mathrm{HClO}_{3}$ molecule, we did not optimize the geometry, but rather used a geometry given by J. S. Francisco and S. P. Saunders, J. Phys. Chem. 100, 573 (1996), which was obtained on an MP2 level with a greatly augmented basis set. On this level, " $\mathrm{HOClO}_{2}$ "' is the favored isomer. For the calculation of the wave function, the same (RHF/6-311G**) level was used.

${ }^{37}$ I. Mayer, Chem. Phys. Lett. 97, 270 (1983). 